\title{
Aspirin and bleeding peptic ulcers in the elderly
}

\author{
Gail Faulkner, P Prichard, K Somerville, M J S Langman
}

\begin{abstract}
A case-control study was performed to determine whether aspirin confers a similar risk of bleeding from gastric or duodenal ulcers in the elderly as nonaspirin, non-steroidal anti-inflammatory drugs. The intake of analgesics in 230 patients with bleeding ulcers aged 60 and over and in hospital and community controls matched for age and sex was examined. Those who had taken aspirin were between two and three times more likely to be admitted to hospital with bleeding ulcers. This increased risk was not accounted for by aspirin taken for indigestion or by concurrent use of non-aspirin, non-steroidal anti-inflammatory drugs. A similar effect was not seen for paracetamol.

When aspirin and other non-steroidal antiinflammatory drugs were considered together the overall risk attributed to the drugs suggested that these drugs may be responsible for over a third of admissions for bleeding peptic ulcers in the elderly.
\end{abstract}

\section{Introduction}

We have found that bleeding from gastric or duodenal ulcers in patients aged 60 and over is strongly associated with the use of non-aspirin, non-steroidal anti-inflammatory drugs. ${ }^{1}$ Aspirin has also been associated with gastrointestinal bleeding, particularly in acute erosive disease, ${ }^{2-7}$ and regular intake of high doses has been associated with the occurrence of uncomplicated gastric (but not duodenal) ulcer. ${ }^{8-11}$ Because most deaths from bleeding peptic ulcer occur in elderly patients we set out to determine whether aspirin confers a similar risk of ulcer bleeding as other non-steroidal anti-inflammatory drugs in this age group. We examined our earlier data to determine whether there was a relation between bleeding from duodenal or gastric ulcers in the elderly and use of nonsteroidal anti-inflammatory drugs.

\section{Subjects and methods}

We considered for inclusion in the study all patients referred to Nottingham University and City Hospitals between April 1983 and March 1985 with haematemesis or melaena. A bleeding gastric or duodenal ulcer was diagnosed endoscopically in 406 of the patients referred. We questioned 327 of these patients, including 230 of the 290 aged 60 and over, on personal details, medical history, and use of drugs. If they had taken analgesics we asked them why they had taken them, which they had taken, and for how long. The 230 patients aged 60 or over were matched for age and sex with hospital and community controls. All 230 hospital controls and 207 of the 230 community controls consented to the study and were questioned, according to the same questionnaire, by the interviewer who had questioned the index patients. Information on the use of analgesics related to the day of the interview for community controls and to the day of the event that had caused admission to hospital for patients with bleeding ulcers and hospital controls.

Matched relative risks (and 95\% confidence intervals) were estimated by the Mantel-Haenszel test applied to matched pair data, and significance was tested by McNemar's test. ${ }^{12}$ Unmatched estimates of relative risk and attributable risk (with $95 \%$ confidence intervals) were those suggested by Fleiss. ${ }^{13}$ All probability values were two tailed.
A spurious correlation between use of aspirin and bleeding ulcers was possible for several reasons: aspirin might have been taken for symptoms related to the bleeding or to relieve pain caused by the ulcer; use of aspirin may be associated with use of other nonsteroidal anti-inflammatory drugs; and histories of aspirin use may differ considerably between patients who have previously had an ulcer and those who have not, although patients who had had an ulcer previously would probably have reduced their intake on medical advice. To take account of these potential biases we firstly made parallel comparisons between the use of aspirin and paracetamol in patients and controls. There is no evidence to suggest that paracetamol causes upper gastrointestinal bleeding yet it is used similarly to aspirin. Any increased risk associated with paracetamol would therefore probably reflect drug use for a bleeding lesion and not that paracetamol caused the bleeding. Secondly, we compared data for different durations of use of analgesics. Thirdly, we made comparisons excluding patients and controls taking aspirin for indigestion and those also taking a nonaspirin, non-steroidal anti-inflammatory drug.

\section{Results}

Table I shows that, depending on the age group, just under a third to just over a half of the patients with bleeding duodenal or gastric ulcers had taken aspirin or other non-steroidal anti-inflammatory drugs in the previous week. Those aged 60 and over were particularly likely to have taken non-aspirin, non-steroidal anti-inflammatory drugs. Table II shows that over twice as many patients had taken aspirin in the preceding week as either hospital or community controls, the differences being highly significant.

The relative risk of bleeding associated with aspirin was significantly greater for use in the previous 24 hours and in the previous week than for regular use (table III). By contrast, no clear trend was associated with use of paracetamol. Exclusion of subjects who had also taken non-aspirin, non-steroidal antiinflammatory drugs and those who had taken aspirin for indigestion did not greatly change the relative risks

TABLE I-Numbers of patients with bleeding peptic ulcers who had taken aspirin and other non-steroidal anti-inflammatory drugs (NSAIDs) in week before event causing admission according to age

\begin{tabular}{lcrrrrr}
\hline & \multicolumn{5}{c}{ Age (years) } \\
\cline { 2 - 7 } & $15-29$ & $30-44$ & $45-59$ & $60-74$ & $\geqslant 75$ \\
\hline Aspirin & 6 & 11 & 8 & 25 & 15 \\
Other NSAIDs & 1 & 1 & 6 & 37 & 30 \\
Aspirin and other NSAIDs & 8 & 21 & 1 & 11 & 2 \\
No NSAIDs & $7(47)$ & $13(38)$ & $15(31)$ & $73(51)$ & $47(54)$ \\
\hline Total (\%) who had taken aspirin & \\
$\quad$ and other NSAIDs & & & & \\
\hline
\end{tabular}

TABLE II-Matched relative risks attributed to intake of aspirin in week before admission in patients with bleeding ulcers compared with matched controls

\begin{tabular}{|c|c|c|c|}
\hline & Cases & $\begin{array}{l}\text { Hospital } \\
\text { controls }\end{array}$ & $\begin{array}{l}\text { Community } \\
\text { controls }\end{array}$ \\
\hline \multirow{4}{*}{$\begin{array}{l}\text { No who had taken aspirin } \\
\text { No who had not taken aspirin } \\
\chi^{2} \text { Value } \\
\text { Matched relative risk ( } 95 \% \\
\text { confidence interval) }\end{array}$} & 53 & 24 & 18 \\
\hline & 177 & 206 & 189 \\
\hline & & $10 \cdot 2$ & \\
\hline & & $2.2(1.4$ to 3.6$)$ & $2.9(1.6$ to 5.2$)$ \\
\hline
\end{tabular}


TABLE III-Unmatched relative risks (95\% confidence intervals) associated with taking aspirin and paracetamol

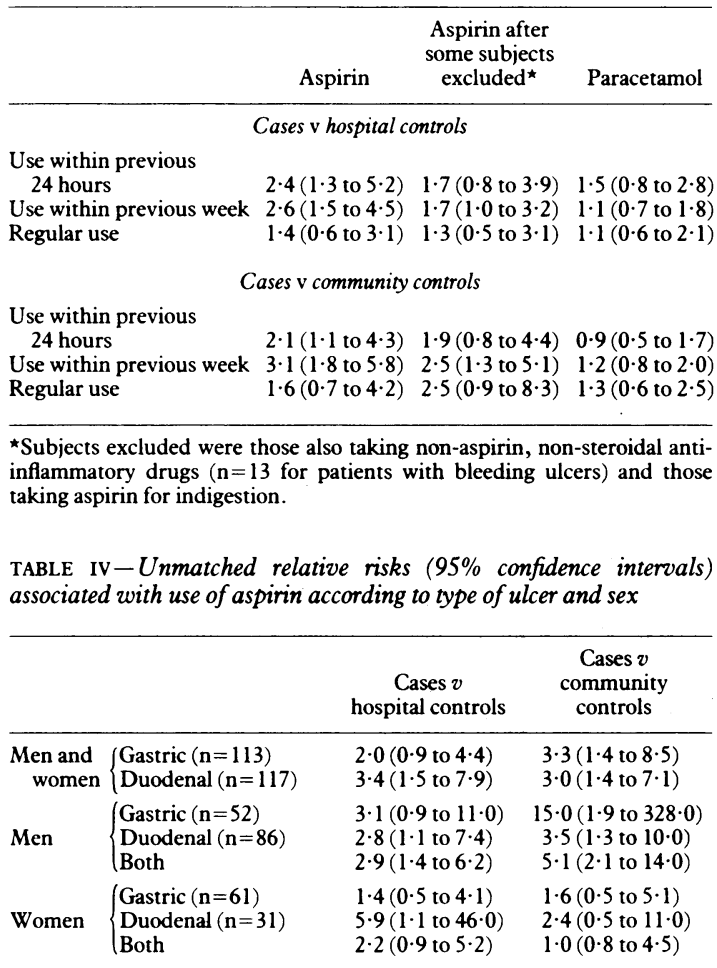

TABLE $\mathrm{v}-$ Reasons for taking aspirin and paracetamol. Numbers in parentheses are numbers of subjects who also took non-aspirin, nonsteroidal anti-inflammatory drugs

\begin{tabular}{|c|c|c|c|c|}
\hline & \multicolumn{3}{|c|}{ Aspirin } & \multirow[b]{2}{*}{$\begin{array}{l}\text { Paracetamol } \\
\quad \text { (cases) }\end{array}$} \\
\hline & Cases & $\begin{array}{l}\text { Hospital } \\
\text { controls }\end{array}$ & $\begin{array}{l}\text { Community } \\
\text { controls }\end{array}$ & \\
\hline Colds and flu & $12(2)$ & 3 & 1 & $4(1)$ \\
\hline Headache & $9(2)$ & 5 & 5 & $5(1)$ \\
\hline Indigestion & $10(2)$ & & & $4(1)$ \\
\hline Osteoarthritis & $4(2)$ & $3(2)$ & $4(1)$ & $16(10)$ \\
\hline Rheumatoid arthritis & $2(1)$ & & & $2(2)$ \\
\hline \multirow{3}{*}{$\begin{array}{l}\text { Transient ischaemic attacks } \\
\text { or circulation problems } \\
\text { Other }\end{array}$} & & & & \\
\hline & 5 & 4 & 3 & 1 \\
\hline & $11(3)$ & 9 & $5(1)$ & $15(8)$ \\
\hline
\end{tabular}

associated with use of aspirin, although risks were slightly lower for those who had taken the drug within the preceding 24 hours or week.

Patients with bleeding ulcers were more likely to have taken aspirin whether those presenting with indigestion $(n=106)$ or those who did not have indigestion $(n=124)$ were considered: 20 with indigestion and 30 without indigestion had taken aspirin. When those who had used aspirin regularly (at least once a week for the previous month) were excluded risks were higher, at 4.0 (confidence interval 1.9 to 9.5 ) and $3.4(1.6$ to $7 \cdot 4$ ) compared with hospital and community controls respectively. Table IV shows that risks associated with use of aspirin were generally increased for both men and women and for gastric and duodenal ulcer, although the figures were variable, probably because of the small numbers studied.

In the whole study population the attributable risk (the proportion of disease directly attributable) for use of aspirin in the previous week was 0.14 (95\% confidence interval 0.09 to 0.19 ) when patients with bleeding ulcers were compared with hospital controls and $0 \cdot 16$ $(0 \cdot 12$ to $0 \cdot 21)$ when the patients were compared with community controls. The values were similar when those who had taken non-aspirin, non-steroidal antiinflammatory drugs or those who had taken aspirin for indigestion were excluded. The combined attributable risk for aspirin and other non-steroidal anti- inflammatory drugs, but excluding aspirin taken for indigestion, was 0.35 when patients were compared with both hospital and community controls (confidence intervals 0.27 to 0.42 and 0.28 to 0.42 respectively). When the patients with bleeding ulcers taking either aspirin or another non-steroidal antiinflammatory drug were considered and compared with hospital and community controls the proportion of their disease attributable to the drug was 0.71 and 0.70 respectively.

Just over half of all the patients and controls who had taken aspirin had taken the soluble type, and a wide variety of preparations were taken by the remainder. The unmatched relative risks for intake of soluble aspirin were $2 \cdot 4(1.2$ to 5.0$)$ and $2 \cdot 8(1.3$ to 6.2$)$ when patients with ulcers were compared with hospital and community controls respectively. These results were similar to those for all varieties of aspirin combined.

Among those who had taken aspirin in the previous week nine (five patients with bleeding ulcers, two hospital controls, and two community controls) had taken the drug prophylactically for transient ischaemic attacks (table V). Of these, three of the patients and none of the controls had taken substantially more than $300 \mathrm{mg}$ daily.

\section{Discussion}

In the United Kingdom the overall incidence of complications of peptic ulcer, as measured by the rate of perforation, is declining; in the elderly, however, little change has occurred among men, while among women the incidence has increased considerably. ${ }^{15}$ Uncontrolled studies have suggested a strong association between intake of non-aspirin, non-steroidal antiinflammatory drugs and the occurrence of perforated or bleeding ulcers, and we previously argued from our case-control data that such drug intake probably accounted for about a fifth of all cases of bleeding ulcers in those aged 60 and over, and the same is probably true for perforated ulcers.'

Data for aspirin are more difficult to interpret. In the United Kingdom most non-aspirin, non-steroidal antiinflammatory drugs are available only on prescription, but most aspirin is taken without prescription: over $80 \%$ of our patients obtained it without prescription. In these circumstances direct comparisons between the proportions of aspirin users among patients with bleeding ulcers and among controls may be misleading because of failure to distinguish between intake of aspirin for symptoms associated with the bleeding lesion, such as dyspepsia, and intake that is likely to cause bleeding. Thus in part of our analysis we excluded patients and controls who had taken aspirin for indigestion and also studied use of paracetamol for comparison. Similarly, because the analgesic intake of hospital controls may well be high because they are ill we also studied community controls as their intake may more truly reflect that of patients with bleeding ulcers. In the event no differences emerged in the use of aspirin between hospital and community controls. A potential source of bias was that we did not question 60 of the 290 patients eligible for the study. General practice records showed, however, that their intake of non-aspirin, non-steroidal anti-inflammatory drugs was about the same as that of the patients we did study, suggesting that their intake of aspirin was also similar.

Previous studies have reported a consistently higher proportion of aspirin users among patients with upper gastrointestinal haemorrhage than controls, ${ }^{2 \cdot 7}$ although when considering peptic ulcers they have generally not shown a strong association. It has also been suggested that any association may be spurious because of use of aspirin for abdominal symptoms. We found, however, that less than $20 \%$ of the patients with 
bleeding ulcers who had taken aspirin the week before presentation had done so because of indigestion. Excluding these reduced the strength of the association of aspirin with bleeding peptic ulcer, but the risk for use in the previous 24 hours and in the previous week remained significantly raised. Because of the association between bleeding peptic ulcer and non-aspirin, non-steroidal anti-inflammatory drugs we determined whether use of these drugs affected our results for aspirin. Excluding patients and controls who had taken these drugs did not substantially alter the association. We therefore conclude that aspirin probably causes peptic ulcer bleeding in the elderly.

We cannot be sure, however, whether aspirin (or other non-steroidal anti-inflammatory drugs) cause existing ulcers to bleed or whether they cause acute mucosal injury which bleeds. The second possibility is plausible because anti-inflammatory drugs can cause acute gastric and duodenal injury with ulceration, ${ }^{16} 17$ and the risk is higher for short term than regular use of aspirin. Adaptation of the mucosa may occur with continued intake, which might explain the decreased association of bleeding ulcers with long term use. On the basis of the present data and our recent findings aspirin and other non-steroidal anti-inflammatory drugs seem to be responsible for about $35 \%$ of admissions for bleeding peptic ulcers of patients aged 60 and over and non-aspirin, non-steroidal anti-inflammatory drugs seem to be responsible for the greater proportion of these. In elderly people taking aspirin or other non-steroidal anti-inflammatory drugs who are admitted to hospital with bleeding from a gastric or duodenal ulcer there is at least a $70 \%$ chance that the drug caused the bleeding.

Our data do not allow us to distinguish between risks associated with different forms of aspirin or to say whether enteric coated or low dose forms, often used to prevent transient ischaemic episodes, are likely to be safe. We can, however, roughly calculate the risks of using aspirin. If the data obtained in our community controls are assumed to apply to the population of the United Kingdom then about $9 \%$ of the 11.5 million people aged 60 and over take aspirin in any one week and just over one million take on average four or five aspirins weekly. About 30000 patients have haematemesis and melaena necessitating admission to hospital, and if our data are typical half of these patients have gastric or duodenal ulcers and two thirds of these, or about 10000 in all, are aged 60 and over. This averages out at about 200 admissions each week. We previously calculated that non-aspirin, non- steroidal anti-inflammatory drugs had an attributable risk of 0.22 and caused 60 cases of bleeding a year in the Nottingham community; extrapolation to the United Kingdom as a whole suggests that they cause 2000 cases a year. ${ }^{1}$ The risk increased to 0.35 when aspirin was also taken into account, suggesting that among the 10000 patients aged 60 and over with bleeding ulcers the bleeding is caused by aspirin or other non-steroidal anti-inflammatory drugs in 3000 cases and by aspirin alone in 1000 . Spread over a year this averages out to 20 cases a week. When related to the total population taking aspirin (roughly one million, each taking four or five tablets weekly) this suggests that about one in every 250000 aspirins taken is associated with bleeding and, assuming a $10 \%$ mortality, one in 2.5 million leads to death.

We thank our colleagues for permission to interview their patients and Boots Company for financial help.

1 Somerville K, Faulkner G, Langman M. Non-steroidal anti-inflammatory drugs and bleeding peptic ulcer. Lancet 1986;i:462-4.

2 Alvarez AS, Summerskill WHJ. Gastrointestinal haemorrhage and salicyclates. Lancet 1958;ii:920-5.

3 Allibone A, Flint FJ. Bronchitis, aspirin, smoking and other factors in the aetiology of peptic ulcer. Lancet 1958;ii:179-82.

4 Parry DJ, Wood PHN. Relationship between aspirin taking and gastroduodenal haemorrhage. Gut 1967;8:301-7.

5 Coggon D, Langman MJS, Spiegelhalter D. Aspirin, paracetamol, and haematemesis and melaena. Gut 1982;23:340-4

6 Langman MJS. Epidemiological evidence for the association of aspirin and acute gastrointestinal bleeding. Gut 1970;11:627-34.

7 Belcon MC, Rooney PJ, Tugwell P. Aspirin and gastrointestinal haemorrhage: a methodologic assessment. $\mathcal{f}$ Chronic Dis 1985;38:101-11.

8 Gilles M, Skyring A. Gastric ulcer, duodenal ulcer, and gastric carcinoma: case-control study of certain social and environmental factors. Med $\mathcal{F}$ Aust 1968;ii:1132-7.

9 Duggan JM. Aspirin in chronic gastric ulcer: an Australian experience. Gut 1976;17:378-84.

10 Piper DW, McIntosh JH, Ariotti DE, Fenton BH, MacLennan R. Analgesic ingestion and chronic peptic ulcer. Gastroenterology 1981;80:427-32.

11 McIntosh JH, Byth K, Piper DW. Environmental factors in aetiology of chronic gastric ulcer: a case control study of exposure variables before the first symptoms. Gut 1985;26:789-98.

12 Breslow NE, Day NE. Statistical methods in cancer research. Vol 1 . The analysis of case-control studies. Lyons: International Agency for Research on Cancer 1980.

13 Fleiss JL. Statistical methods for rates and proportions. 2nd ed. New York: Wiley, 1981:56-82.

14 Last JM, ed. A dictionary of epidemiology. Oxford: Oxford University Press, 1983.

15 Walt R, Katschinski B, Logan R, Ashley J, Langman M. Rising frequency of ulcer perforation in elderly people in the United Kingdom. Lancet 1986; 489-92.

16 Lanza FL, Rover JR, Nelson RS, Chen TT, Seckman CE, Rack MF. The effects of ibuprofen, indomethacin, aspirin, naproxen, and placebo on the gastric mucosa of normal volunters. A gastroscopic and photographic gastric mucosa of normal volu
study. Dig Dis Sci 1979;24:823-8.

17 Hoftiezer JW, O'Laughlin JL, Ivey KJ. The effects of 24 hours of aspirin, Bufferin, paracetamol and placebo on normal human gastroduodenal Bufferin, paracetamol and

18 Levy M. Aspirin use in patients with major upper gastrointestinal bleeding and peptic-ulcer disease. N Engl F Med 1974;290:1158-62.

(Accepted 16 August 1988)

\section{Castration at birth}

\section{Samuel George}

26 Ince Road, Walton on Thames, Surrey KT12 5BJ Samuel George, MRCOG, registrar in obstetrics and gynaecology
A great variety of injuries to bone and soft tissue have been attributed to the trauma of manipulation during vaginal breech delivery, especially during breech extraction. To my knowledge castration has not been reported before.

\section{Case report}

A 25 year old Asian woman having her first baby booked at 16 weeks' gestation. At 32 weeks the presentation was noted to be breech, and this persisted despite external cephalic version at 34 weeks. Lateral $x$ ray pelvimetry at 36 weeks showed an anteroposterior diameter of the inlet, mid-cavity, and outlet of
$12.5 \mathrm{~cm}, 12.0 \mathrm{~cm}$, and $11.5 \mathrm{~cm}$ respectively. The baby felt average in size, and the breech was engaged.

The patient came to the labour ward at 40 weeks with spontaneous rupture of the membranes and was having strong regular contractions. Vaginal examination showed a breech presentation at the level of the ischial spines, well applied to the cervix. The cervix was dilated to $3 \mathrm{~cm}$, almost fully effaced, soft, and central. She had an epidural anaesthetic, and she reached full dilatation in six hours. Her progress in the second stage of labour was also normal, and the breech (in a left sacroanterior position) with extended legs was visible at the introitus after half an hour. A generous right posterolateral episiotomy was performed quickly as the patient was pushing and the breech was distending the perineum. Soon afterwards a pearly greyish pink spherical structure about $1.5 \mathrm{~cm}$ in diameter fell out of the vagina into the obstetrician's hand. The patient kept pushing, and another similar structure was passed out. There was no undue bleeding. 